\title{
Population and breast cancer patients' analysis reveals the diversity of genomic variation of the BRCA genes in the Mexican population
}

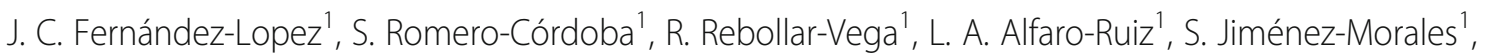 \\ F. Beltrán-Anaya ${ }^{1}$, R. Arellano-Llamas' ${ }^{1}$ A. Cedro-Tanda' ${ }^{1}$ M. Rios-Romero ${ }^{1}$, M. Ramirez-Florencio ${ }^{1}$, V. Bautista-Piña ${ }^{2}$, \\ C. Dominguez-Reyes ${ }^{2}$, F. Villegas-Carlos ${ }^{2}$, A. Tenorio-Torres ${ }^{2}$ and A. Hidalgo-Miranda ${ }^{1 *}$ (D)
}

\begin{abstract}
Interpretation of variants of unknown significance (VUS) in genetic tests is complicated in ethnically diverse populations, given the lack of information regarding the common spectrum of genetic variation in clinically relevant genes. Public availability of data obtained from high-throughput genotyping and/or exome massive parallel sequencing (MPS)-based projects from several thousands of outbred samples might become useful tools to evaluate the pathogenicity of a VUS, based on its frequency in different populations. In the case of the Mexican and other Latino populations, several thousands of samples have been genotyped or sequenced during the last few years as part of different efforts to identify common variants associated to common diseases. In this report, we analyzed Mexican population data from a sample of 3985 outbred individuals, and additional 66 hereditary breast cancer patients were analyzed in order to better define the spectrum of common genomic variation of the BRCA1 and BRCA2 genes. Our analyses identified the most common genetic variants in these clinically relevant genes as well as the presence and frequency of specific pathogenic mutations present in the Mexican population. Analysis of the 3985 population samples by MPS identified three pathogenic mutations in BRCA1, only one population sample showed a BRCA1 exon 16-17 deletion by MLPA. This resulted in a basal prevalence of deleterious mutations of $0.10 \%$ (1:996) for BRCA1 and 11 pathogenic mutations in BRCA2, resulting in a basal prevalence of deleterious mutations of $0.276 \%$ (1:362) for BRCA2, combined of $0.376 \%$ (1:265). Separate analysis of the breast cancer patients identified the presence of pathogenic mutations in 18\% (12 pathogenic mutations in 66 patients) of the samples by MPS and 13 additional alterations by MLPA. These results will support a better interpretation of clinical studies focused on the detection of BRCA mutations in Mexican and Latino populations and will help to define the general prevalence of deleterious mutations within these populations.
\end{abstract}

Keywords: BRCA, Breast cancer, Populations, Germline, Genetic testing

\footnotetext{
* Correspondence: ahidalgo@inmegen.gob.mx

'Laboratorio de Genómica del Cáncer, Instituto Nacional de Medicina

Genómica, Perfiérico Sur, 4809, Arenal Tepepan, 14610 Mexico City, CP,

Mexico

Full list of author information is available at the end of the article
}

(C) The Author(s). 2019 Open Access This article is distributed under the terms of the Creative Commons Attribution 4.0 International License (http://creativecommons.org/licenses/by/4.0/), which permits unrestricted use, distribution, and reproduction in any medium, provided you give appropriate credit to the original author(s) and the source, provide a link to the Creative Commons license, and indicate if changes were made. The Creative Commons Public Domain Dedication waiver (http://creativecommons.org/publicdomain/zero/1.0/) applies to the data made available in this article, unless otherwise stated. 


\section{Introduction}

BRCA1 and BRCA2 might represent two of the most characterized genes in the human genome due to their association with hereditary cancer syndromes. However, the complete spectrum of BRCA genetic variation among ethnically diverse populations has not been fully described. Germline mutations in these genes are mainly associated to familial breast and ovarian cancer and more recently with pancreatic and prostate cancer. In addition to the identification of subjects with a higher risk of developing cancer in a familial setting, detection of deleterious germline $B R C A$ mutations is also required for the selection of treatment with PARP inhibitors, which can induce synthetic lethality in cancer cells in the presence of deleterious BRCA mutations. Currently, treatment with PARP inhibitors are approved for patients with deleterious or suspected deleterious germline BRCA mutations with HER2-negative metastatic breast cancer, for patients with hormone receptor-positive breast tumors that have been treated or are not susceptible to receive endocrine therapy [1], and in patients with advanced ovarian cancer who have been treated with three or more prior lines of chemotherapy [2].

The prevalence of combined mutations in the totality of the coding region of these genes vary between different countries and ethnic groups, being approximately $0.3 \%$ in Caucasian women in the USA and $2.5 \%$ in Jewish women living in Israel or in the USA. A recent paper focused on the analysis of exome sequencing-based screening for BRCA1/2 among adult biobank participants identified a higher frequency of pathogenic/likely pathogenic mutations than previous reports, identifying a 1:180 prevalence of deleterious mutations and suggesting that compared with previous clinical care, exome sequencing-based screening identified five times as many individuals with pathogenic or likely pathogenic BRCA1/ 2 variants [3].

The prevalence of mutations in Latin American populations has not yet been fully defined. In Mexico, a limited number of analyses in the BRCA1 and BRCA2 genes have been carried out using different methods [4-10]. These studies have identified the presence of pathogenic germline mutations in $28 \%$ of the patients with ovarian cancer and $15 \%$ of the patients with breast cancer, without any selection for family history. The percentage of BRCA1 mutations in women diagnosed with triple negative breast tumors, without any selection for familial cancer, increases up to $28 \%$. In patients with ovarian cancer, without any selection for familial cancer, the BRCA1 ex9-12del mutation was detected in $33 \%$ of the cases, supporting the notion that this is a founder mutation in Mexico $[8,10]$.

A recent review about mutations in the BRCA genes identified a clear founder effect in several Latin American populations, including Mexico (BRCA1 ex9-12del), Brazil
(BRCA1 5382insC y BRCA2 c.156_157insAlu), and Colombia (BRCA1 3450del4, A1708E, y BRCA2 3034del4), as well as in Latino population from southern California (BRCA1 185delAG, IVS5+1G>A, S955x, y $\mathrm{R} 1443 \mathrm{x})$. The differences in the frequency and type of $B R C A$ mutations in Latin America have been associated with the admixture dynamics in each specific population and with the differences in the proportions of ancestral components resulting from the admixture processes over time [11].

However, the analysis of a much higher number of samples is necessary in order to define the frequency of pathogenic mutations and to define the whole spectrum of common genetic variation in clinically relevant genes in the Mexican population [12]. With the advent of massive parallel sequencing and the reduction of costs for sequencing-based diagnostic panels, this situation is already improving. Nonetheless, it will take time to have enough clinical samples to define the population-wide spectrum of common variation and to define a better threshold to evaluate pathogenicity based on allele frequency, as recommended by the American Colleague of Medical Genetics and Genomics and the Association for Molecular Pathology. To define this threshold, it is necessary to have population-based genotyping information, in order to determine if the allele frequency of a particular variant is "higher" than expected for the disorder, a situation that provides strong evidence to consider the variant benign [13]. Fortunately, thanks to the public availability of data obtained from high-throughput genotyping and/or massive parallel exome sequencing projects from several thousands of outbred samples (ExAC [14], 1000 genomes [15]), we can analyze the presence of specific variants in different populations in order to compare how common they are and to evaluate their potential pathogenicity depending on their allele frequency.

In the case of the Mexican and other Latino populations, several thousands of samples have been genotyped or sequenced during the last few years as part of different efforts to identify common variants associated to common diseases, such as diabetes (The Slim Initiative in Genomic Medicine for the Americas (SIGMA) T2D Consortium) [16-18]. This information has led to the identification of diabetes-related variants enriched in the Mexican population, but has also provided population-based frequencies of common genetic variants throughout the genome, information which can be used to define the spectrum of common genetic variation in clinically relevant genes.

In this report, we analyzed Mexican population data from a sample of 3985 outbred individuals, and additional 66 hereditary breast cancer patients were analyzed in order to better define the spectrum of common genomic variation of the BRCA1 and BRCA2 genes. Our 
analyses identified the most common genetic variants in these clinically relevant genes as well as the presence and frequency of specific pathogenic mutations present in the Mexican outbred population and corroborated the presence and frequency of pathogenic mutations in hereditary breast cancer patients. These results will support a better interpretation of clinical studies focused on the detection of BRCA mutations in Mexicans and Latino populations and will help to define the general prevalence of deleterious mutations within these populations.

\section{Materials and methods Studied population}

All the procedures and protocols were reviewed and approved by the Ethics and Research Committee of the National Institute of Genomic Medicine (INMEGEN) and were compliant with the Helsinki declaration. BRCA1 and BRCA2 genetic variants data included in this study were obtained from 3985 outbred individuals from two sources: (1) the SIGMA Type 2 Diabetes Whole Exome Sequencing Project database and (2) open population samples from the Mexican Genome Diversity Project (MGDP), where the BRCA1 and BRCA2 genes were analyzed by massive parallel sequencing and multiplex ligation-dependent probe amplification (MLPA).

Additionally, 66 samples from women with breast cancer with history of familial cancer were also analyzed.

\section{Population samples, source 1: SIGMA Type 2 Diabetes Whole Exome Sequencing Project}

There are 3842 unrelated individuals from the Slim Initiative in Genomics Medicine for the Americas Type 2 Diabetes Whole-Exome Sequence Project (SIGMA Type 2 Diabetes). Data is deposited in the type 2 diabetes knowledge portal [18].

\section{Population samples, source 2: Mexican Genome Diversity Project}

There are 143 unrelated anonymous women with no associated phenotype, which were collected as part of the Mexican Genome Diversity Project (MGDP), as described by Silva-Solezzi, et al [19]. These samples were selected from the Mexican States of Campeche, Zacatecas, Sonora, Yucatán, Tamaulipas, Guerrero, Guanajuato, and Veracruz and are considered the "Mestizo" population (admixed). We also included samples from the Amerindian Tepehuano group, from Durango, the Zapoteco group from Oaxaca, and Mayas from Campeche.

\section{Women with breast cancer and history of familial cancer} Sixty-six samples from breast cancer patients with familial cancer history, which were identified by a clinical geneticist, were included in the study after informed consent at the Instituto de Enfermedades de la Mama FUCAM, AC.

Both the MGDP and the breast cancer patients were analyzed for $B R C A 1$ and $B R C A 2$ mutations by massive parallel sequencing at the National Institute of Genomic Medicine in Mexico City. Variants and frequencies of the BRCA1 and $B R C A 2$ genes from individuals from the SIGMA project were identified as described in references $[16,17]$.

\section{Ancestral components of the SIGMA and MGDP samples}

All data sets had either Mexican or other Latino ancestry based on self-reporting. This was corroborated using principal component analysis of genotype data. The average ancestry proportion of the Native American component in the SIGMA Type 2 Diabetes data set was 0.69. In the MGDP Mestizo samples, the ancestry components were Native American 0.59, European 0.37, and African 0.04. The Amerindian ancestry proportion in the Tepehuano, Zapoteco, and Maya samples was 0.97, 0.02 European, and 0.003 African. Figure 1 shows the principal components analysis (PCA) and ancestral component distribution of the Mexican Genome Diversity Project samples. Additional file 1: Table S1 lists the complete ancestral components of each of these samples.

\section{BRCA1 and BRCA2 massive parallel sequencing analysis} Genomic DNA from blood lymphocytes was purified with the QIAamp DNA Blood Maxi Kit. DNA was quantified and adjusted to a concentration of $5 \mathrm{ng} / \mu \mathrm{L}$ using a Qubit 2.0 Fluorometer (Invitrogen, Waltham, MA, USA) and the Qubit dsDNA HS Assay Kit (Invitrogen). For target enrichment, 50 ng of genomic DNA was amplified using custom primers designed to target all coding exons of BRCA1 and $B R C A 2$, and library preparation was done using the TruSeq HT library preparation kit (Illumina, San Diego, CA, USA) according to the manufacturer's instructions. Pooled libraries were sequenced on a MiSeq sequencer (Illumina) using the MiSeq Reagent Kit v2 (300 cycles). Sequencing data was analyzed using Illumina's BaseSpace TruSeq amplicon tools V3.0 and the variants that passed all quality controls were annotated using ClinVar and BRCAExchange.

\section{MLPA}

Exonic deletions and duplications affecting the $B R C A$ genes were detected on genomic DNA using the multiplex ligation-dependent probe amplification (MLPA) commercial kits from MRC-Holland, Amsterdam, The Netherlands, according to the manufacturer's recommendations. The Coffalyser software (V.140721.1958) was used for data analysis.

\section{Annotation of variants}

ClinVar [20] (28/02/18) and BRCAexhange (http:// brcaexchange.org/ version 19/10/17) data were used to 


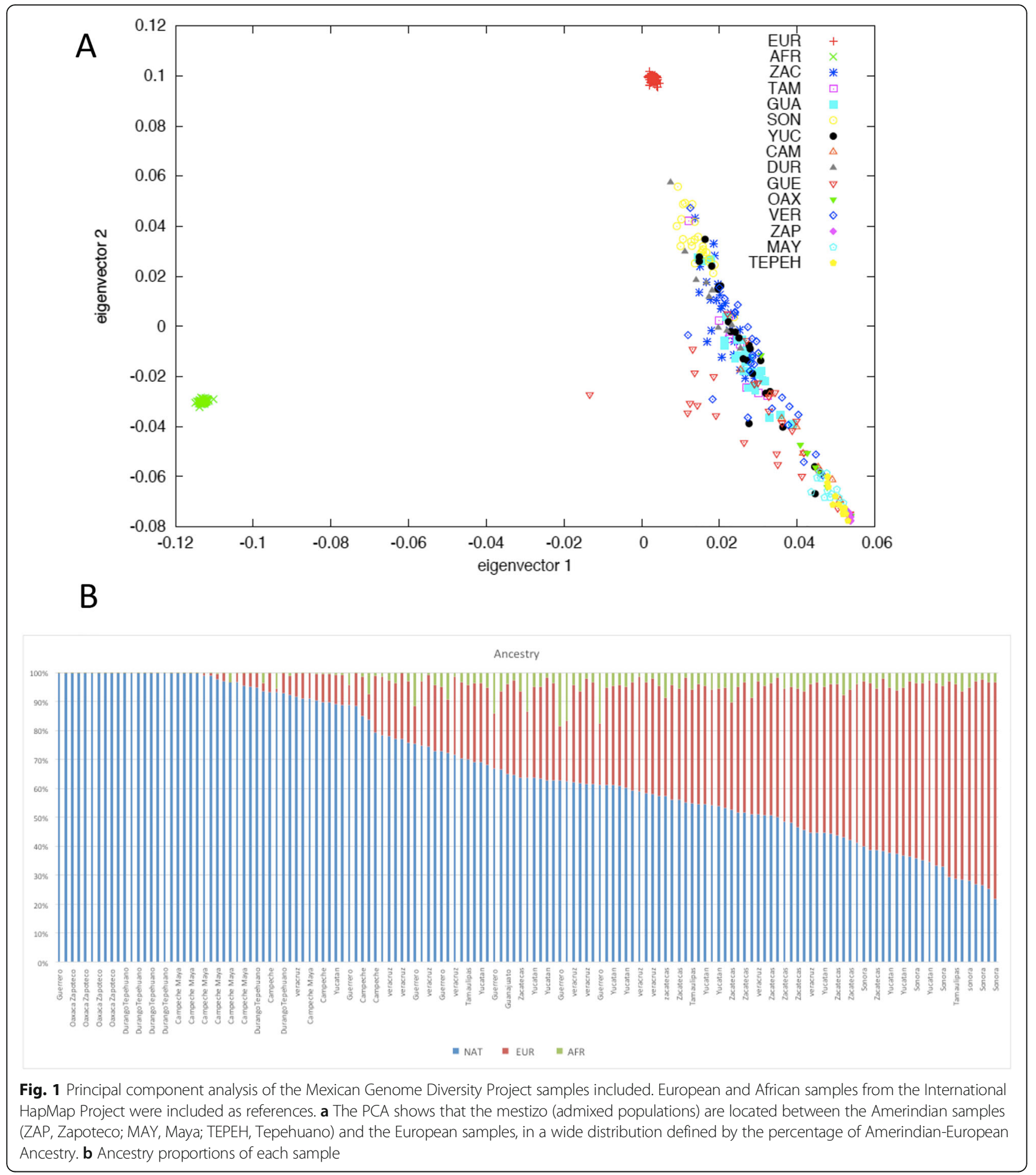

annotate all variants. Even though the BRCA exchange data includes allele frequency from ExAC, this version does not include the total number of Latino samples reported in the current version of ExAC, so we updated this information directly from the ExAC portal (http:// exac.broadinstitute.org/downloads version 1.0 02/27/17).
Only variants that passed the genotyping quality metrics in ExAC were included in the analysis.

\section{Availability of data and materials}

The datasets supporting the conclusions of this article are included within the article and its additional files. 
These data include all the variants detected in the population samples analyzed in this study.

\section{Results \\ Frequency of BRCA mutations in the Mexican outbred population samples}

From a sample consisting of 3985 population samples (143 sequenced in this study and 3842 from the SIGMA study), we identified 15 pathogenic mutations (3 detected by massive parallel sequencing and 1 by MPLA in $B R C A 1$ and 11 in BRCA2, all detected by sequencing), resulting in a population frequency of deleterious mutations of $0.10 \%$ (1:996) for BRCA1 and $0.276 \%$ (1:362) for $B R C A 2$, combined of $0.376 \%$ (1:265).

Table 1 shows the 15 pathogenic mutations identified in the open population samples for BRCA1 and BRCA2.

Identification of BRCA variants in the Mexican population We identified 160 variants in $B R C A 1$ and 274 variants in $B R C A 2$ based on the analysis of 3842 Mexican population samples from the SIGMA Diabetes database.

Regarding the 160 variants in BRCA1, 52 were benign, 33 were benign-likely benign, 23 had conflicting interpretations of pathogenicity, 18 were of uncertain significance, and only 3 were pathogenic (NM_007294.3:c.5123C>A, NM_0072 94.3:c.4327C >T, NM_007294.3:c.3648dupA). Twenty-nine variants are not found in ClinVar and have not been yet reviewed by the ENIGMA consortium, out of these, one affects a splice region and eight are missense mutations. One mutation (NM_007294.3:c.1729G>C) is predicted as possibly damaging by polyphen (score 0.77 ). Of the 160 variants, 69 have only been reported in the Latino population from ExAC and might represent Latino-specific variants.

Regarding BRCA2, 274 variants were found, of which, 143 were missense, 51 synonymous, two generated a stop gain, one a start loss, five were in splice regions, one in non-coding transcript exons, 54 were in introns, one was an inframe insertion, one an inframe deletion, eight frameshift and four were in the $3^{\prime}$ and 5 ' regions.

Eleven of the mutations found in BRCA2 in the population dataset were classified as pathogenic by the ENIGMA consortium or ClinVar. Thirty-seven variants were not in ClinVar and have not been reviewed by ENIGMA. Eight of these were missense and three (NM_000059.3 c.2635T $>C$; NM_000059.3 c.6416A $>$ T; NM_000059.3 c.8816A>G) were predicted as probably damaging by polyphen (score 0.996).

From a population standpoint, 121 of the 174 variants detected in the Mexican subjects from SIGMA project have not been observed in other groups and are also private of the Latino population in ExAC. These seemingly Latino private variants include six of the ten pathogenic mutations in BRCA2, a situation that is confirmed by the ethnicity report of the ClinVar submitters reporting some of these mutations. They are all low-frequency mutations which are present as heterozygotes in one individual out of the approx. 3842 samples analyzed, except for one (rs80359775) which is present as heterozygote in six individuals.

Regarding variants of unknown significance in $B R C A 2$, 106 of the 274 variants were classified as either "uncertain significance" or "conflicting interpretations of pathogenicity." Nineteen were missense mutations classified as possibly or probably deleterious by Polyphen or SIFT and showed a low-allele frequency on all populations suggesting they might probably represent pathogenic variants.

Additional file 2: Table S2 and Additional file 3: Table S3 show the complete list of variants detected in all samples, together with their annotation.

Open population from Mexican genome diversity Project The 143 open population samples from the MGDP did not present any pathogenic mutation, except for one sample with a BRCA1 x16-17del deletion (Additional file 4: Table S4). Variants with the highest allele frequency identified in the SIGMA database were also between the most common identified in the MGDP sample. Additional file 4: Table S4 shows the MLPA results.

\section{Mutation analysis in breast cancer patients}

Sixty-six samples with a suspected history of familial cancer were sequenced. Massive parallel sequencing identified pathogenic mutations in 12 samples (18\%, Table 2), two of these were also found in the open population samples (NM_007294.3:c.4327C>T; NM_007294.3:c.3648dupA). Seven additional mutations classified as uncertain significance were classified as "probably damaging" by polyphen.

\section{MLPA}

As described before, 49 population samples were analyzed for $B R C A 1$, only one population sample showed a deletion in exons 16-17 (BRCA1 ex16-17del). For $B R C A 2$, MLPA analysis did not find alterations in any of the 55 open population samples analyzed.

In 55 patients with history of familial breast cancer, the founder BRCA1 ex9-12del was identified in two samples and a third sample with familial history showed a BRCA1 ex12deletion.

In the 64 samples with history of familial breast cancer, BRCA2 exon 1 deletions were found in three cases, deletions of exon 11 in one case, deletion of exon 23 in two cases, exon 17 and exon 26 were deleted in one case each, and two samples presented BRCA2 ex22-24 deletions. MLPA results from these cases are presented in Additional file 4: Table S4.

Pathogenic mutations found in our dataset were identified as such in the BRCA Exchange database, based on the clinical significance defined by the ENIGMA 


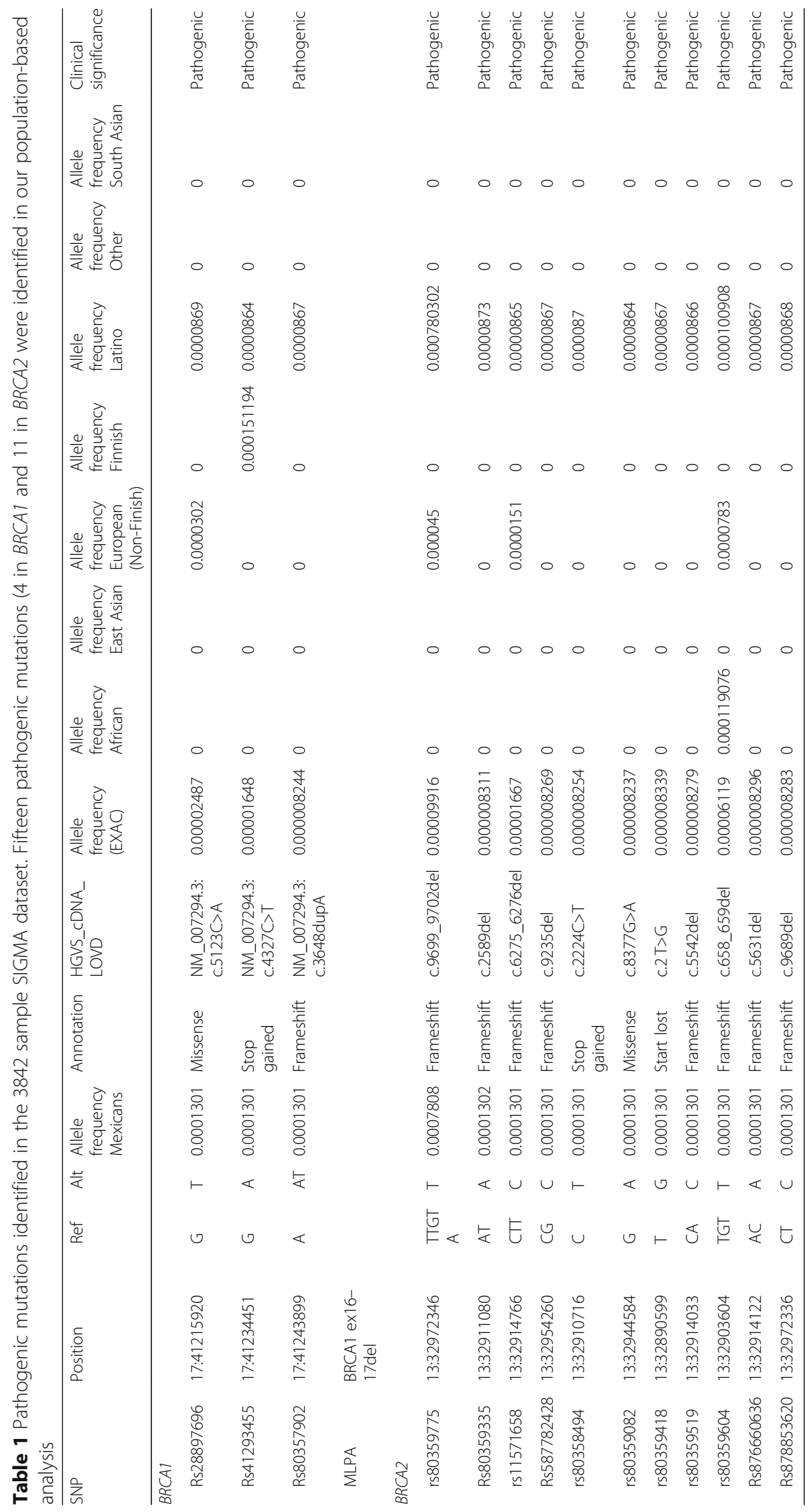


Table 2 Pathogenic mutations identified in the breast cancer patients

\begin{tabular}{|c|c|c|c|c|c|c|c|c|}
\hline Sample ID & RS & GENE & POS & REF & ALT & $\begin{array}{l}\text { Clinical_significance } \\
\text { _ENIGMA }\end{array}$ & HGVS_cDNA & REFERENCE \\
\hline FUCAM29 & rs80357520 & BRCA1 & 41243787 & TTA & $\mathrm{T}$ & Pathogenic & c.3759_3760delTA & Villareal-Garza 2015 [8] \\
\hline FUCAM50 & rs41293455 & BRCA1 & 41234451 & G & A & Pathogenic & $c .4327 C>T$ & McKean-Cowdin 2005 [24] \\
\hline FUCAM53 & rs80357382 & BRCA1 & 41258474 & $\mathrm{~T}$ & C & Pathogenic & $c .211 A>G$ & Rebbek 2016 [25] \\
\hline FUCAM56 & rs80357780 & BRCA1 & 41245250 & $\mathrm{ACT}$ & A & Pathogenic & c.2296_2297delAG & Weitzel 2005 [26] \\
\hline FUCAM65 & rs80357902 & BRCA1 & 41243899 & A & AT & Pathogenic & c.3648dupA & Lecarpentier 2012 [27] \\
\hline FUCAM75 & rs80357842 rs80357889 & BRCA1 & 41243686 & CCTCA & C & Pathogenic & c.3858_3861delTGAG & Kwong 2016 [28] \\
\hline FUCAM77 & rs80357914 & BRCA1 & 41276044 & A & ACT & Pathogenic & c.68_69delAG & Bolton 2012 [29] \\
\hline FUCAM1 & rs777107618 rs80359380 & BRCA2 & 32911755 & C & CT & Pathogenic & c.3264dupT & Susswein 2016 [29] \\
\hline FUCAM36 & rs41293513 & BRCA2 & 32937507 & A & G & Pathogenic & $C .8168 A>G$ & Guidugli 2013 [30] \\
\hline FUCAM40 & rs80359660 & BRCA2 & 32930683 & G & GC & Pathogenic & c.7556dupC & Borg 2010 [31] \\
\hline FUCAM48 & rs80359082 & BRCA2 & 32944584 & G & A & Pathogenic & $c .8377 G>A$ & Guidugli 2013 [30] \\
\hline FUCAM310 & rs80359082 & BRCA2 & 32944584 & G & A & Pathogenic & c. $8377 \mathrm{G}>\mathrm{A}$ & Guidugli 2013 [30] \\
\hline \multicolumn{9}{|l|}{ MLPA } \\
\hline FUCAM33 & & BRCA1 & & & & & Ex9-12del & \\
\hline FUCAM11 & & BRCA1 & & & & & Ex9-11del & \\
\hline FUCAM30 & & BRCA1 & & & & & Ex12del & \\
\hline FUCAM84 & & BRCA2 & & & & & Ex1del & \\
\hline FUCAM85 & & BRCA2 & & & & & Ex23del & \\
\hline FUCAM98 & & BRCA2 & & & & & Ex1del & \\
\hline FUCAM100 & & BRCA2 & & & & & Ex17del & \\
\hline FUCAM102 & & BRCA2 & & & & & Ex23del & \\
\hline FUCAM9 & & BRCA2 & & & & & Ex22-24del & \\
\hline FUCAM10 & & BRCA2 & & & & & Ex22-24del & \\
\hline FUCAM25 & & BRCA2 & & & & & Ex11del & \\
\hline FUCAM41 & & BRCA2 & & & & & Ex26del & \\
\hline FUCAM47 & & BRCA2 & & & & & Ex1del & \\
\hline
\end{tabular}

consortium. The population frequencies were obtained from our data from the Mexican population and from other populations from the ExAC database. In both cases, the observed frequency of this allele is very low. However, the pathogenicity of these mutations is supported in several ways, including their report by several submitters, a good segregation with disease, deleterious effects on protein structure, analyzed in silico, and a high posterior probability of pathogenicity from multifactorial likelihood analysis.

\section{Discussion}

Precise results interpretation of genomic testing is of paramount importance, both for the clinical management of the patients and to avoid unnecessary stress derived from an uncertain result. Erroneous interpretation of genetic data, such as when a patient is incorrectly informed that one of his or her variants is causal when in fact it is benign, have important adverse consequences for the patients and for their families.

A better interpretation of these analyses requires the inclusion of populations of diverse ethnical backgrounds, both through access to the tests themselves and also as part of scientific efforts aimed to describe human genomic diversity and its role in human disease. Several examples clearly show that this lack of representation already represents an important clinical problem for the interpretation of genetic tests [21], resulting in what has been called a "double disparity" where access to testing is limited and the interpretation of results are complicated by the lack of data from populations with a non-European background [22].

In Mexico, access to genetic testing for hereditary breast and ovarian cancer is still not widely available, limiting the amount of patient-derived data necessary to refine interpretation of their results. Fortunately, data generated from the research front is helping to mitigate 
this problem. In the last years, high-throughput genotyping and whole exome/genome sequencing efforts have included outbred samples from diverse ethnical populations, offering the possibility to contrast the frequency of suspicious variants against open population frequencies. These efforts include the Mexican genome diversity project $[19,23]$ and, more recently, exome sequencing projects aimed to the identification of common variants associated to diabetes and other diseases [16-18]. Recent studies have shown that this exome-based population approach might be more efficient in the identification of the frequency of pathogenic or likely pathogenic $B R C A 1 / 2$ mutations, being able to identify five times as many individuals with deleterious mutations compared to studies focused on selected populations in the clinical care [3].

Based on this data, in this paper, we describe the spectrum of common genomic variation in the $B R C A$ genes in the Mexican population. Our analyses allowed us to identify variants that are enriched in the Mexican and Latino populations and to identify the identity and frequency of pathogenic mutations present in open population samples.

From a sample consisting of 3985 population samples (143 sequenced in this study and 3842 from the SIGMA study), we identified 15 pathogenic mutations (3 detected by massive parallel sequencing and 1 by MPLA in BRCA1 and 11 in BRCA2, all detected by sequencing), resulting in a population frequency of deleterious mutations of $0.10 \%$ (1:996) for BRCA1 and $0.276 \%$ (1:362) for BRCA2, combined of $0.376 \%$ (1:265). This is similar to what has been reported for population frequency carriers in ExAC without The Cancer Genome Atlas (TCGA) samples: $0.15 \%$ (1:646) for BRCA1 and $0.26 \%$ for BRCA2 (1:390; combined $0.41 \% 1: 243)$. In 2016, the total population in Mexico was of 127.5 million, escalating the frequency of mutations, resulting in approximately 481,132 carriers of deleterious BRCA mutations among the Mexican population.

In order to evaluate the potential pathogenicity of variants with uncertain clinical significance, based on population-based data, we reviewed both the frequency and filtering allele frequency of the seven VUS detected in our breast cancer patients. The low frequency of an allele might be a criterion suggesting variant pathogenicity, but since frequency alone is not sufficient to define association with disease, we also reviewed their statistical threshold to filter them out if they are too common in the population to be associated with disease, based on the ExAC data (filtering $\mathrm{AF}$ in the ExAC browser). Four variants were filtered: rs80358861 (in European non-Finish), rs80359018 (Latino), rs80357323 (African), rs80358877 (Latino), but still, for two additional variants (rs80358947, rs80358621), this approach was not possible, since they are not described ExAC and have not yet been reviewed by ENIGMA). Sixty-seven additional variants were identified in the breast cancer patients which have not been described in BRCA Exchange and are not present in ExAC and might represent private polymorphisms.

Our sample collection reflects the genomic diversity of the Mexican population, based on the sample distribution in our PCA analysis, and we did not observed enrichment of particular variants regardless of the state of origin of the sample. We expected to see an enrichment of European-associated variants in the Northern states of Mexico (Sonora) or in samples with a higher percentage of European ancestral component. This suggests that the distribution of $B R C A$ variants among the Mexican population might not differ significantly throughout different regions of the country. In fact, our dataset is particularly enriched in samples with a higher percentage of Amerindian ancestry (75\% of Amerindian ancestral component on average); this would more likely represent variants which are enriched in in the Amerindian population, and this is supported by the observation that most of the variants detected in our population-based analysis were only present on the Latino population of ExAC.

In conclusion, our analyses allowed us to better define the common genomic variation of the $B R C A$ genes in the Mexican open population, identifying specific pathogenic mutations and allowing the first calculation of the mutation burden in these clinically relevant genes in Mexico. Given the observed enrichment of these mutations the Latino population, our data will also be helpful to improve the interpretation of $B R C A 1$ and $B R C A 2$ mutation tests in other Latin American countries.

\section{Additional files}

Additional file 1: Table S1. Ancestry information of all Mexican Genome Diversity Project samples included in the sequencing analysis. (XLSX 34 kb)

Additional file 2: Table S2. All variants detected in BRCA1 in the 3842 SIGMA samples, together with their annotation. (XLSX 254 kb)

Additional file 3: Table S3. All variants detected in BRCA2 in the 3842 SIGMA samples, together with their annotation. (XLSX $455 \mathrm{~kb}$ )

Additional file 4: Table S4. MLPA analysis of the Breast cancer samples. (XLSX 37 kb)

\section{Acknowledgements}

This work was funded by a grant from Astra Zeneca Mexico (ESR-16-12517) granted to AHM. We thank all the patients for their participation in the study.

\section{Authors' contributions}

JFL, SRC, LAR, AHM, and SJM, did the data analysis. RALL performed MLPA experiments and data analysis. RRV, FBA, and ACD did the sample processing. MRR, and MRL did the sample collection. VBP, CDR, FVC, and ATT are responsible for the patient identification. AHM contributed in the study design and project coordination. All authors discussed the findings and participated in writing of the final manuscript; all authors agreed on the final version of the manuscript. 


\section{Competing interests}

AHM received a grant from Astra Zeneca Mexico for this paper. The other authors declare that they have no competing interests.

\section{Publisher's Note}

Springer Nature remains neutral with regard to jurisdictional claims in published maps and institutional affiliations.

\section{Author details}

'Laboratorio de Genómica del Cáncer, Instituto Nacional de Medicina Genómica, Perfiérico Sur, 4809, Arenal Tepepan, 14610 Mexico City, CP, Mexico. ${ }^{2}$ Instituto de Enfermedades la Mama FUCAM, Avenida El Bordo 100, Santa Ursula Coapa, 04980 Mexico City, CP, Mexico.

Received: 9 July 2018 Accepted: 18 December 2018

Published online: 10 January 2019

\section{References}

1. Robson M, et al. Olaparib for metastatic breast cancer in patients with a germline BRCA mutation. N Engl J Med. 2017;377(6):523-33.

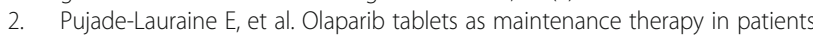
with platinum-sensitive, relapsed ovarian cancer and a BRCA1/2 mutation (SOLO2/ENGOT-Ov21): a double-blind, randomised, placebo-controlled, phase 3 trial. Lancet Oncol. 2017;18(9):1274-84.

3. Manickam $\mathrm{K}$, et al. Exome sequencing-based screening for brca1/2 expected pathogenic variants among adult biobank participants. JAMA Network Open. 2018;1(5):e182140

4. Calderon-Garciduenas AL, et al. Clinical follow up of Mexican women with early onset of breast cancer and mutations in the BRCA1 and BRCA2 genes. Salud Publica Mex. 2005:47(2):110-5.

5. Ruiz-Flores P, et al. BRCA1 and BRCA2 mutation analysis of early-onset and familial breast cancer cases in Mexico. Hum Mutat. 2002;20(6):474-5.

6. Vaca-Paniagua $F$, et al. Full-exon pyrosequencing screening of BRCA germline mutations in Mexican women with inherited breast and ovarian cancer. PLoS One. 2012;7(5):e37432.

7. Vidal-Millan S, et al. Mutational analysis of BRCA1 and BRCA2 genes in Mexican breast cancer patients. Eur J Gynaecol Oncol. 2009:30(5):527-30.

8. Villarreal-Garza C, et al. The prevalence of BRCA1 and BRCA2 mutations among young Mexican women with triple-negative breast cancer. Breast Cancer Res Treat. 2015;150(2):389-94.

9. Weitzel JN, et al. Prevalence and type of BRCA mutations in Hispanics undergoing genetic cancer risk assessment in the southwestern United States: a report from the clinical cancer genetics community research network. J Clin Oncol. 2013;31(2):210-6.

10. Villarreal-Garza $C$, et al. Significant clinical impact of recurrent BRCA1 and BRCA2 mutations in Mexico. Cancer. 2015;121(3):372-8

11. Ossa CA, Torres D. Founder and recurrent mutations in BRCA1 and BRCA2 genes in Latin American countries: state of the art and literature review. Oncologist. 2016;21(7):832-9.

12. Narod SA. Screening for BRCA1 and BRCA2 mutations in breast cancer patients from Mexico: the public health perspective. Salud Publica Mex. 2009;51(Suppl 2):s191-6.

13. Richards S, et al. Standards and guidelines for the interpretation of sequence variants: a joint consensus recommendation of the American College of Medical Genetics and Genomics and the Association for Molecular Pathology. Genet Med. 2015;17(5):405-24.

14. Lek $M$, et al. Analysis of protein-coding genetic variation in 60,706 humans. Nature. 2016;536(7616):285-91.

15. Genomes Project $C$, et al. A global reference for human genetic variation. Nature. 2015;526(7571):68-74.

16. Consortium, S.T.D, et al. Association of a low-frequency variant in HNF1A with type 2 diabetes in a Latino population. JAMA. 2014;311(22):2305-14.

17. Consortium, S.T.D, et al. Sequence variants in SLC16A11 are a common risk factor for type 2 diabetes in Mexico. Nature. 2014;506(7486):97-101.

18. Portal, T.D.K., Type 2 diabetes knowledge Portal. 2018.

19. Silva-Zolezzi l, et al. Analysis of genomic diversity in Mexican Mestizo populations to develop genomic medicine in Mexico. Proc Natl Acad Sci U S A. 2009:106(21):8611-6

20. NCBI, ClinVar. 2018.

21. Manrai AK, et al. Genetic misdiagnoses and the potential for health disparities. N Engl J Med. 2016;375(7):655-65.
22. Gerhard GS, Fisher SG, Feldman AM. Genetic testing for inherited cardiac diseases in underserved populations of non-European ancestry: double disparity. JAMA Cardiol. 2018;3(4):273-4.

23. Moreno-Estrada A, et al. Human genetics. The genetics of Mexico recapitulates Native American substructure and affects biomedical traits. Science. 2014;344(6189):1280-5.

24. McKean-Cowdin $\mathrm{R}$, et al. BRCA1 variants in a family study of AfricanAmerican and Latina women. Hum Genet. 2005;116(6):497-506.

25. Rebbeck TR, et al. Inheritance of deleterious mutations at both BRCA1 and BRCA2 in an international sample of 32,295 women. Breast Cancer Res. 2016;18(1):112.

26. Weitzel JN, et al. Prevalence of BRCA mutations and founder effect in highrisk Hispanic families. Cancer Epidemiol Biomark Prev. 2005;14(7):1666-71.

27. Lecarpentier J, et al. Variation in breast cancer risk associated with factors related to pregnancies according to truncating mutation location, in the French national BRCA1 and BRCA2 mutations carrier cohort (GENEPSO). Breast Cancer Res. 2012;14(4):R99.

28. Kwong A, et al. Comprehensive spectrum of BRCA1 and BRCA2 deleterious mutations in breast cancer in Asian countries. J Med Genet. 2016;53(1):15-23

29. Bolton $\mathrm{KL}$, et al. Association between BRCA1 and BRCA2 mutations and survival in women with invasive epithelial ovarian cancer. JAMA. 2012; 307(4):382-90.

30. Guidugli $L$, et al. A classification model for BRCA2 DNA binding domain missense variants based on homology-directed repair activity. Cancer Res. 2013;73(1):265-75.

31. Borg A, et al. Characterization of BRCA1 and BRCA2 deleterious mutations and variants of unknown clinical significance in unilateral and bilateral breast cancer: the WECARE study. Hum Mutat. 2010;31(3):E1200-40.

\section{Ready to submit your research? Choose BMC and benefit from:}

- fast, convenient online submission

- thorough peer review by experienced researchers in your field

- rapid publication on acceptance

- support for research data, including large and complex data types

- gold Open Access which fosters wider collaboration and increased citations

- maximum visibility for your research: over $100 \mathrm{M}$ website views per year

At $\mathrm{BMC}$, research is always in progress.

Learn more biomedcentral.com/submissions 